\title{
О риск-нейтральном подходе ценообразования реальных опционов
}

\author{
Дранев Ю.Я. ${ }^{24}$
}

Оченка компаний и проектов с помощью метода реальных опщионов (все более популярного в развитых странах) в значительной мере зависит от правильного выбора модели. Предложенная в данной работе семимартингальная модель позволяет учитывать нестачионарные и скачкообразные изменения финансовых показателей, в отличие от традиционной модели Блэка-Шолиа. Более того, в модели допускается, что изменения зависят от значений параметров в предыдущие моменты времени. Проблема заключается в том, что такая модель может быть не полной, и риск-нейтральное состояние для такой модели не определяется однозначно. В этом случае иена опџиона определяется путем максимизации функции полезности, как это описал Калсен [Kallsen, 1999]. В заключение продемонстрирована возможность применения численных методов для ценообразования реальных опционов в неполных семимартингальных моделях.

JEL: $G 30$

Ключевые слова: реальные опџฺионы, неполная модель, функциия полезности

\section{Введение}

По сравнению с традиционным методом дисконтирования денежных потоков основным преимуществом оценки инвестиционных проектов с помощью метода реальных опционов является возможность учитывать управленческую гибкость менеджмента. С момента введения Майерсом в 1977 году [Mayers, 1977] понятия реального опциона значительное число исследований было посвящено этой концепции. Ключевыми вопросами применения метода реальных опционов являются как поиск оптимальной стохастической модели, отражающей изменение параметров инвестиционного проекта в условиях неопределенности, так и определение в рамках выбранной модели цен реальных опционов, возникающих в процессе реализации этого проекта.

С учетом некоторых особенностей проблемы ценообразования реальных опционов тесно связаны с ценообразованием опционов на финансовом рынке. Модель Блэка-Шолца [Black, Scholes, 1973] и полученная ими знаменитая формула для цены европейского коллопциона на акцию в условиях непрерывного времени, получила широкое применение и для расчета цен реальных опционов. Помимо целого ряда упрощений основным недостатком модели Блэка-Шолца является предположение о том, что цена актива $S(t)$ является геометрическим броуновским движением, т.е. удовлетворяет стохастическому дифференциальному уравнению вида:

(1) $\frac{d S}{S}=b d t+\sigma d W \quad c$ решением $\quad S=S(0) \exp \left(\left(b-\sigma^{2} / 2\right) t+\sigma W\right)$,

где $W$ - процесс броуновского движения, а $b$ и $\sigma$ параметры сдвига и стандартного отклонения.

Однако геометрическое броуновское движение, впервые предложенное для описания поведения цен на финансовом рынке в условиях непрерывного времени в работе Самуэлсона

\footnotetext{
${ }^{24} \mathrm{Ph} . \mathrm{D}$, старший преподаватель кафедры экономики и финансов фирмы, ГУ ВШЭ. 
[Samuelson, 1964], не вполне точно моделирует изменение стоимости активов как на финансовых рынках, так и в инвестиционных проектах. Действительно, скачкообразные изменения, зависимые или нестационарные приращения являются характерными для процессов, наблюдаемых на финансовом рынке, и при этом никак не согласуются с предположением о том, что приращение логарифмов изменения цен является броуновским движением вокруг некоторого тренда (сдвига). В дальнейшем был опубликован целый ряд работ, посвященных смягчению условий и обобщениям модели Блэка-Шолца (например, Aace [Aase, 1988], Бэндорф-Нильсен [Barndorff-Nielsen, 1977] и др.).

В основе ценообразования опционов лежат два взаимосвязанных подхода. Первый подход состоит в том, что цена опциона С при определенных условиях может быть найдена через значение хеджирующего портфеля (портфеля-копии для реальных опционов), состоящего из одного (или нескольких) базового актива $S$ и безрискового актива $B$ (например, государственной облигации или банковского депозита): $\pi_{0}(t) B+\pi_{l}(t) S(t)$. Причем $\left(\pi_{0}, \pi_{1}\right)$ является хеджирующей стратегией и определяется через решение стохастического дифференциального уравнения с некоторыми краевыми условиями. Тогда цена европейского опциона будет равна значению хеджирующего портфеля в момент $T$ исполнения опциона (в частности, формула Блэка-Шолца). При этом для нахождения цен реальных опционов базовый актив в портфеле-копии часто замещается торгуемым активом, со значениями, в некотором смысле близкими к значениям базового актива или корреллирующими с ними.

Двумя важнейшими условиями, при которых работает метод портфеля копии, являются: во-первых, безарбитражность, т.е. невозможность получить безрисковый доход; а во-вторых, полнота, т.е. существование портфеля-копии для любого дериватива. Если условие безарбитражности для инвестиционных проектов представляется легко выполнимым, так как можно предположить, что в реальных условиях невозможно без риска перепродать нерыночный актив, то обеспечить выполнение условия полноты для проектов с активами, у которых либо нет торгуемых аналогов, либо торгуемые аналоги не отражают все риски проекта, практически невозможно. У проекта помимо рыночного риска часто присутствует внутренний (идиосинкразический) риск. В этом случае для ценообразования опционов может использоваться так называемый риск-нейтральный подход.

Риск-нейтральный подход связан с концепцией эффективного рынка и заключается в следующем. Вероятностное пространство, на котором заданы стохастические процессы, моделирующие изменение цен активов, преобразуется с помощью непрерывной замены вероятностных мер так, что в новом «риск-нейтральном» вероятностном пространстве цены активов становятся «нейтральными к риску». В том числе выполнено условие: средние будущие значения цен активов не зависят от риска. Другими словами, в новом рискнейтральном вероятностном пространстве цены активов, дисконтированные по безрисковой ставке, становятся мартингалами (условное матожидание такого процесса в момент времени $S(t)$, при условии, что заданы значения при $0<s<t$, равно значению в момент $s: E(S(t) \mid S(s)$, $0<s<t)=S(s)$ ). Свойство мартингальности можно еще сформулировать так: среднее значение процесса в будущем периоде, при условии известной истории процесса до текущего момента, равно значению процесса в текущий момент времени. При этом вероятностную меру, соответствующую вероятностному пространству, в котором цены активов становятся мартингалами, называют мартингальной. В случае, когда мартингальная мера единственная, она является риск-нейтральной.

Риск-нейтральный подход позволил значительно обобщить модель Блэка-Шолца. Так, семимартингалы, которые представляют собой сумму процесса ограниченной вариации и мартингала, являются одним из наиболее широких классов случайных процессов, для которых исследованы вопросы ценообразования опционов с использованием рискнейтрального подхода: например, Харрисон, Плиска [Harrison, Pliska, 1981]. Жакод, Ширяев [Jacod, Shiryaev. 1987] подробно описали свойства семимартингалов, их характеристики и интегрирование по семимартингалам. В класс семимартингалов помимо броуновского 
движения попадают процессы Леви с независимыми стационарными приращениями и более общие скачкообразные процессы с зависимыми приращениями.

Включение скачкообразного компонента в процесс, описывающий изменение цены активов, особенно популярно для моделей, используемых в ценообразовании реальных опционов. Так, Тригеоргис [Trigeorgis, 1996] добавил пуассоновский процесс к геометрическому броуновскому движению для описания потока возникающих конкурирующих проектов. Достаточно много работ посвящено скачкообразному влиянию регулятивных мер на активы и на реальные опционы проекта. Особенно часто такие ограничения возникают в энергетике и энергосберегающих проектах. Например, Лиин и Хуан [Lin, Huang, 2009]. Баро и Беллами [Barrieu, Bellamy, 2007] исследовали бессрочные реальные опционы в семимартингальной модели, где процессы не обладали свойствами Леви. Большое количество работ посвящено реальным опционам с необратимыми инвестициями.

Конечно, существуют хорошо изученные процессы, которые не попадают в класс семимартингалов. Таким процессом является фрактальное броуновское движения со свойством самоподобия. Однако модели, использующие самоподобие, относятся больше к теории поведенческих финансов на фондовом рынке, и они практически неприменимы для описания реальных активов в инвестиционных процессах.

Так же как и для метода портфеля-копии, важнейшими свойствами, определяющими применимость риск-нейтрального подхода в семимартингальных моделях, является безарбитражность и полнота. Делбаен и Шахермаер [Delbaen, Schachermayer, 1998] доказали наличие связи между безарбитражностью и существованием риск-нейтральных мер для случая непрерывного времени в семимартингальной модели. Харрисон и Плиска [Harrison, Pliska, 1981] определили, что при некоторых условиях для семимартингалов полнота, т.е. представимость опциона через портфель-копию, эквивалентна единственности мартингальной меры $P^{*}$ (ее риск-нейтральности). В случае выполнения условий безарбитражности и полноты, с учетом дисконтирования цен активов по безрисковой ставке, цена европейского опциона со страйком $K$ и временем исполнения $T$ определяется как:

$C=E_{P *}(\max (S(T)-K), 0)$.

Экономический смысл матожидания по риск-нейтральной мере заключается в том, что менеджмент, принимая решение об исполнении опциона в момент времени $T$, уже осведомлен обо всех рисках в этот момент времени, и при подсчете цены опциона надо учитывать это обстоятельство. Вспоминая, что матожидание эквивалентно интегрированию по вероятностной мере, и опуская строгие математические выкладки, цену опциона можно записать так:

где $Z=d P^{*} / d P$ называется плотностью замены вероятностных мер и является производной Радона Никодима абсолютно непрерывной меры $P^{*}$ относительно меры $P$. Смысл умножения опциона на $Z$ экономически аналогичен дисконтированию по ставке,

$$
\left.C=\int(\max (S(T)-K), 0) d P^{*}=\int(\max (S(T)-K), 0) \frac{d P^{*}}{d P} d P=E(Z \max (S(T)-K), 0)\right),
$$

скорректированной с учетом риска. Такой подход принципиально отличается от подхода дисконтированных денежных потоков, при которых нет управленческой гибкости, а значит, дисконтирование происходит по отражающей рыночные риски ставке (определяемой, например, с помощью САРМ).

Если не выполнено условие полноты, т.е. если невозможно подобрать портфель копию из активов на финансовом рынке, цены реальных опционов могут быть только лишь приближены состоящими из торгуемых активов портфелями, хеджирующими рыночные риски (но не внутренние риски проекта). В этом случае мы не можем говорить о рискнейтральности, так как мартингальных мер становится больше одной и выбор мартингальной меры, а значит, и цены опциона, связан с риском. Говорят еще, что невозможна полная трансформация риска реального опциона в риски торгуемых активов. При этом цены опционов, соответствующие различным мартингальным мерам, принадлежат 
интервалу на прямой, вне которого менеджмент проекта, не стремящийся к риску, предпочтет исполнению реальных опционов безрисковые операции на финансовом рынке с торгуемыми активами. Этот интервал в литературе еще называют безарбитражными границами (например, Пуйо [Руо, 2008]). Таким образом, встает вопрос выбора «оптимальной», или «равновесной», цены реального опциона в рамках безарбитражных границ, а значит, и соответствующей мартингальной меры, с учетом максимизации полезности. Этому вопросу посвящено большое количество работ. Так, Швейцер [Schweizer. 1991] определил и исследовал минимальную мартингальную меру. Позднее Швейцер [Schweizer, 1996] изучал мартингальные меры, процесс плотности которых имеет наименьшую дисперсию. Калсен и Ширяев [Kallsen, Shiryaev, 2002] обобщили мартингальную меру, полученную с помощью преобразования Долеан на случай семимартингалов. Критерий оптимальности через минимизацию энтропии был получен Фрителли [Fritelli, 2000]. Гол и Ручендорф [Goll, Ruchendorf, 2001] предложили выбирать наиболее близкую в некоторой метрике оптимальную мартингальную меру. Были рассмотрены и другие методы выбора мартингальных мер.

Калсен [Kallsen, 1999] предложил максимизировать функции ожидаемой полезности при ценообразовании опционов в семимартингальных моделях. Для каждого инвестиционного проекта функция полезности может быть выбрана различными способами, в зависимости от расположенности к риску (risk aversion). Например, выбор мартингальной меры с наименьшей дисперсией плотности снижает риск завышения стоимости реальных опционов проекта. Исходя из выбранной функции полезности, можно определить плотность мартингальной меры, а значит, и оптимальную стоимость реального опциона проекта.

В работе анализируется ценообразование опционов в модели инвестиционного проекта с несколькими активами, цены которых дисконтированы по безрисковой ставке и являются семимартингалами специального вида со скачкообразной составляющей. Подобная модель не является в общем случае полной, т.е. нет возможности найти портфель-копию из торгуемых активов, учитывающих все риски проекта. Поэтому для определения цены реального опциона на расширение путем покупки дополнительного актива через время $T$ рассматриваются различные критерии оптимальности выбора мартингальной меры, которая максимизирует соответствующую функцию полезности. Приведенные ниже примеры демонстрируют применение методики для получения явной формулы для цены реального опциона на расширение, аналогичной формуле Блэка-Шолца. С помощью метода МонтеКарло демонстрируется, как изменяется процесс, описывающий цену актива, и распределение цены опциона после замены меры на минимальную мартингальную.

\section{Модель и мартингальные меры}

Рассмотрим набор цен активов инвестиционного проекта $S(t)=\left(S_{l}(t), \ldots, S_{m}(t)\right)$. Для удобства вычислений мы предполагаем, что все цены дисконтированы по безрисковой ставке в каждый момент времени. Мы предполагаем, что цена активов не может быть отрицательной, т.е. можно записать $S_{i}=\exp \left(R_{i}\right), i=1, \ldots, m$.

Случайные колебания цен на различные виды сырья и продукции, изменяющиеся постоянные издержки и другие факторы значительно влияют на стоимость активов. Таким образом, инвестиционный проект зависит от большого числа факторов риска (часто большему, чем число активов проекта). Предположим, что в проекте присутствуют $k$ факторов риска $(k \geq m)$. Для описания неопределенностей непрерывного характера, например, связанных с колебаниями цен на сырье в периоды экономической стабильности, рассмотрим $d$-мерное броуновское движение $W=\left(W_{l}, \ldots, W_{d}\right)$. Как уже отмечалось, в инвестиционных проектах часто встречаются неопределенности, связанные с новыми регулятивными нормами, кризисными явлениями в экономике, появлением конкурентов или новых продуктов и т.д. Влияние таких неопределенностей оказывает скачкообразный эффект на денежные потоки, а значит, и на стоимость активов. Для их моделирования 
целесообразно использовать маркированные точечные процессы (см. например, Бремо [Bremaud, 1981]). Итак, рассмотрим $k-d$ процессов $N_{i}$, каждый из которых представляет собой последовательность пар $\left(t_{n}, z_{n}, n \geq 1\right)$, где $t_{n}$ - время $n$-го скачка, а $z_{n}-$ характеризующая размер скачка случайная величина с плотностью распределения $j(t, z)$. Предположим также, что интенсивность скачков задана положительной функцией $\lambda_{j}(t)$.

Каждый из факторов риска, характерных для проекта в целом, по-разному отражается на различных активах проекта. Влияние $j$-го «непрерывного» фактора риска на $i$-й актив будем моделировать с помощью предсказуемого процесса интегрируемой вариации (см. например Жакод, Ширяев [Jacod, Shiryaev, 1987]) $\sigma_{i j}^{W}(t)$. Аналогично влияние $j$-го «скачкообразного» фактора риска на $i$-й актив будем моделировать с помощью предсказуемого процесса интегрируемой вариации $\sigma^{N}{ }_{i j}(t, z)$, который для каждого фиксированного $t$ является монотонной дифференцируемой по $z$ функцией.

Наконец, для описания прогнозируемых темпов роста стоимости актива $S_{i}$ будем использовать предсказуемый процесс $b_{i}(t)$.

Мы предполагаем, что выполнены все условия, обеспечивающие корректное интегрирование по случайным процессам.

Тогда логарифм цены $i$-го актива может быть записан следующим образом:

$$
\log \left(S_{i}(t)\right)=R_{i}(t)=\int_{0}^{t} b_{i}(s) d s+\sum_{j=1}^{d} \int_{0}^{t} \sigma_{i j}^{W}(s) d W_{j}(s)+\sum_{j=1}^{k-d} \int_{0}^{t} \int_{R} \sigma_{i j}^{N}(s, z) d N_{j}(s, z) .
$$

Можно заметить, что если продифференцировать обе части выражения (2), то получится форма записи, эквивалентная (1).

Одной из особенностей функции экспоненты является тот факт, что если процесс $R$ является мартингалом относительно некоторой меры $P^{*}$, то процесс $\exp (R)$ совершенно не обязательно также будет мартингалом. Поэтому запишем цену $i$-го актива в виде $S_{i}=\varepsilon(R \quad i)$, где $\varepsilon$ - экспонента Долеан (см. например, Калсен, Ширяев [Kallsen,Shiryaev 2002]), важнейшим свойством которой является сохранение свойства мартингальности. Введем следующие обозначения:

$$
\begin{aligned}
& €_{i j}^{N}(t, z)=e^{\sigma_{i j}^{N}(t, z)}-1, \quad d q_{j}(t, z)=d N(t, z)-\lambda_{j}(t) \phi_{j}(t, z) d t d z \\
& \mathscr{\epsilon}_{i}(t)=b_{i}(t)+\frac{1}{2} \sum_{j=1}^{d}\left(\sigma_{i j}^{W}(t)\right)^{2}+\sum_{j=1}^{k-d} \lambda_{\mathrm{j}}(\mathrm{t}) \int_{\mathrm{R}}\left(€_{i j}^{N}(t, z)-\sigma_{i j}^{N}(t, z)\right) \phi(t, z) d z
\end{aligned}
$$

Здесь $q_{j}$ - мартингал, получающийся компенсированием маркированного точечного процесса $\mathrm{N}_{\mathrm{j}}$.

Предполагая, что $\sigma \mathrm{ij}^{\mathrm{N}}(\mathrm{t}, \mathrm{z})$ является предсказуемым процессом интегрируемой вариации и, пользуясь свойствами экспоненты Долеан, можно записать:

$$
\AA_{i}(t)=\int_{0}^{t} E_{i}(s) d s+\sum_{j=1}^{d} \int_{0}^{t} \sigma_{i j}^{W}(s) d W_{j}(s)+\sum_{j=1}^{k-d} \int_{0}^{t} \int_{R} \oint_{i j}^{N}(s, z) d q_{j}(s, z) .
$$

Если найти мартингальную меру $P^{*}$, относительно которой все процессы $R{ }_{i}$ станут мартингалами, то из свойства экспоненты Долеан будет следовать, что все процессы $S_{i}$, описывающие изменение цен активов, также станут мартингалами. Согласно Жакоду, Ширяеву [Jacod, Shiryaev, 1987], каждый семимартингал однозначно характеризуется триплетом предсказуемых характеристик $(B, C, v)$, где $B$ - процесс ограниченной вариации, $C$ - характеризует непрерывную мартигальную часть, а $v$ - компенсатор скачкообразной части. Для того чтобы семимартингал относительно меры $P^{*}$ стал мартингалом, его триплет относительно $P^{*}$ должен иметь вид $\left(0, C^{*}, v^{*}\right)$.

Мартингальная мера $P^{*}$ определяется процессом плотности $Z=d P^{*} / d P$. В рассмотренной модели свойство полноты не всегда выполняется, поэтому, вообще говоря, существует семейство мартингальных мер и соответствующих им процессов плотности. Так как $E(Z)=1$, а значит, $Z$ является мартингалом, то мы будем искать процессы плотности в виде $Z=\varepsilon(M)$, где $M-$ квадратично интегрируемый мартингал. Применяя свойства 
триплетов предсказуемых характеристик и обобщенную теорему Гирсанова (см. например, [Ширяев, 1998]), мы получим следующее семейство процессов плотности, соответствующее мартингальным мерам:

$$
Z=\mathcal{E}\left(-\sum_{j=1}^{d} \int_{0}^{t} v_{j}(s) d W_{j}(s)-\sum_{j=1}^{k-d} \int_{0}^{t} \int_{R} \eta_{j}(s, z) d q_{j}(s, z)\right), \text { где } \quad v_{\mathrm{j}}(\mathrm{s}) \quad \text { и } \quad \eta_{\mathrm{j}}(\mathrm{s}, \mathrm{z}), \text { являются }
$$

предсказуемыми процессами с интегрируемой вариацией и удовлетворяют системам линейных уравнений для каждого фиксированного $t$ :

$$
\bar{F}_{i}(t)-\sum_{j=1}^{d} \sigma_{i j}^{W}(t) v_{j}(t)-\sum_{j=1}^{k-d} \lambda_{\mathrm{j}}(\mathrm{t}) \int_{\mathrm{R}} \epsilon_{i j}^{N}(t, z) \eta_{j}(t, z) \phi_{j}(t, z) d z=0, i=1, \ldots, m .
$$

Пусть $k>m$ и $\operatorname{det}\left(\Sigma \Sigma^{t r}\right) \neq 0$, где $\Sigma-$ матрица линейной системы уравнений (5). Тогда система (5) имеет бесконечно много решений. Если предположить, что $\eta_{j}(s, z)<1$, то меры, получаемые с помощью (4), будут вероятностными. В противном случае меры будут со знаком (подробнее см., например, Швейцер [Schweizer, 1996]).

Предположим, что после преобразования меры (4) маркированный точечный процесс $N_{j}$ имеет характеристики: интенсивность $\lambda_{j}^{*}(t)=r_{j}(t) \lambda_{j}(t)$ и плотность распределения

$j_{j}^{*}(t, z)=\psi_{j}(t, z) \quad j(t, z)\left(\right.$ т.е. $\left.\int \psi_{j}(t, z) \quad{ }_{j}(t, z) d z=1\right)$, соответствующие новой мере $P^{*}$. Тогда $\eta_{j}(s, z)=1-r_{j}(t) \psi_{j}(t, z)$. Более того, цена актива имеет вид:

$$
S_{i}(t)=\mathcal{E}\left(\sum_{j=1}^{d} \int_{0}^{t} \sigma_{i j}^{W}(s) d W_{j}^{*}(s)+\sum_{j=1}^{k-d} \int_{0}^{t} \int_{R} \oint_{i j}^{N}(s, z) d q_{j}^{*}(s, z)\right),
$$

где $W_{j}^{*}(t)=W_{j}(t)+\int v_{j}(s) d s-$ броуновское движение относительно мартингальной меры $P^{*}$, а $q_{j}^{*}$ - компенсированный маркированный точечный процесс с характеристиками $\lambda_{j}{ }^{*}(t)$ и $j^{*}(t, z)$.

Решая систему (5) для каждого $t$, находим такие $v_{j}(s), r_{j}(t), \psi_{j}(t, z)$, что $\psi_{j}(t, z)>0$ и выполняется условие $\int \psi_{j}(t, z) j(t, z) d z=1$, которые задают процесс плотности, а значит, и мартингальную меру. Проблема заключается в том, что в общем случае решений у системы (5) бесконечно много. В случае полноты единственность мартингальной меры соответствует выбору стратегии, которая полностью хеджирует все риски. Однако в ситуации, когда нет хеджирующей опцион стратегии, остается риск, связанный с выбором оптимальной в некотором смысле мартингальной меры.

\section{Оптимальные мартингальные меры}

Есть достаточно много способов найти оптимальную мартингальную меру. Так, Швейцер [Schweizer, 1996] предложил минимизировать дисперсию плотности $\operatorname{Var}(Z)=$ $E\left(d P^{*} / d P\right)^{2}-1=E^{*}(Z)-1$. Фрителли [Fritelli, 2000] исследовал свойства меры, минимизирующую энтропию, т.е. $E(Z \log (Z))=E^{*}(\log (Z)$. Было множество других способов оптимизации (например, Калсен и Ширяев [Kallsen, Shiryaev, 2002]).

В своей работе Калсен [Kallsen, 1999] определил общий класс функций полезности $u$ следующим образом:

1) $u$ - дважды дифференцируема;

2) первая и вторая производные - $u^{\prime}$ и $u^{\prime \prime}-$ ограничены, и $u(x) \rightarrow 0$ при $x \rightarrow \infty$;

3) $u(0)=0, u^{\prime}(0)=1$;

4) $u^{\prime}(x)>0$ для всех $x$;

5) $u^{\prime \prime}(x)<0$ для всех $x$.

Основная идея Калсен [Kallsen, 1999] заключается в том, чтобы выбрать стратегию, которая максимизирует ожидаемую полезность прибыли на бесконечно малых отрезках. С помощью такой стратегии можно сконструировать мартингальную меру. В нашем случае будем выбирать стратегию $\pi(t)=\left(\pi_{1}(t), \ldots, \pi_{m}(t)\right)$, максимизирующую ожидаемую полезность прироста логарифма цены актива (локадьную полезность). Применяем результат Калсена. Стратегия $\pi(\mathrm{t})$ находится путем решения линейной системы уравнений для $i=1, \ldots, m$ : 


$$
\begin{gathered}
E_{i}(t)+u^{\prime \prime}(0) \sum_{j=1}^{d} \sigma_{i j}^{W}(t) \sum_{l=1}^{m} \pi_{l}(t) \sigma_{l j}^{W}(t)- \\
-\sum_{j=1}^{k-d} \lambda_{\mathrm{j}}(\mathrm{t}) \int_{\mathrm{R}} \oint_{i j}^{N}(t, z)\left(u^{\prime}\left(\sum_{i=1}^{m} \pi_{i}(t) \biguplus_{i j}^{N}(t, z)\right)-1\right) \phi_{j}(t, z) d z=0 .
\end{gathered}
$$

В случае существования стратегии $\pi(t)$ параметры мартингальной меры $P^{*}$ будут заданы следующими формулами:

$$
\begin{gathered}
v(t)=-u^{\prime \prime}(0) \sum_{i=1}^{m} \pi_{i}(t) \sigma_{i j}^{W}(t), \\
r_{j}(t)=\int_{R} u^{\prime}\left(\sum_{i=1}^{m} \pi_{i}(t) \bigotimes_{i j}^{N}(t, z)\right) \phi_{j}(t, z) d z, \\
\psi_{j}(t, z)=\frac{u^{\prime}\left(\sum_{i=1}^{m} \pi_{i}(t) \bigotimes_{i j}^{N}(t, z)\right)}{\int_{R} u^{\prime}\left(\sum_{i=1}^{m} \pi_{i}(t) \bigotimes_{i j}^{N}(t, z)\right) \phi_{j}(t, z) d z} .
\end{gathered}
$$

Таким образом, выбирается мартингальная мера, соответствующая такой стратегии инвестиций в активы проекта, что на бесконечно малых промежутках времени ожидаемая полезность прироста логарифма оценки проекта максимизируется.

Применяя результат Швейцера [Schweizer, 1996], можно показать, что мартингальная мера с минимальной дисперсией плотности (variance optimal, или минимальная мартингальная) соответствует в нашем случае функции полезности:

$$
u(x)=x-x^{2} / 2 \text {, при } x<1 \text {. }
$$

Другим распространенным способом выбора оптимальной меры является минимизация энтропии плотности $Z \log (Z)$ (см., например, Фрителли [Fritelli, 2000]). Такой мартингальной мере будет соответствовать функция полезности:

$u(x)=1-\exp (-x)$.

Как уже отмечалось, есть много других способов выбора оптимальной мартингальной меры.

\section{Примеры}

\section{1. Пуассоновские процессы}

Предположим, что в рассмотренной модели $N_{j}(j=1, \ldots, k-d)$ являются пуассоновскими процессами с постоянными интенсивностями $\lambda_{j}$. Размеры скачков также не зависят от времени и характеризуются постоянными $\sigma_{i j}^{N}(i=1, \ldots, m)$. Пусть также $\sigma^{W}{ }_{i j}=$ const и $b_{i}=$ const. В этом частном случае семимартингалы $R_{i}(i=1, \ldots, m)$ становятся процессами Леви с независимыми стационарными приращениями:

$$
R_{i}(t)=\log S_{i}(t)=b_{i} t+\sum_{j=1}^{d} \sigma_{i j}^{W} W_{j}(t)+\sum_{j=1}^{k-d} \sigma_{i j}^{N} N_{j}(t) .
$$

Обозначая $d q_{j}=d N_{j}(t)-\lambda_{j} t$ и применяя (3), получим:

$$
\begin{aligned}
& S_{i}(t)=\varepsilon\left(E_{i} t+\sum_{j=1}^{d} \sigma_{i j}^{W} W_{j}(t)+\sum_{j=1}^{k-d}\left(e^{\sigma_{i j}^{N}}-1\right) q_{j}(t)\right), \\
& \bar{F}_{i}(t)=b_{i} t+\frac{1}{2} \sum_{j=1}^{d}\left(\sigma_{i j}^{W}\right)^{2}+\sum_{j=1}^{k-d} \lambda_{\mathrm{j}}\left(e^{\sigma_{i j}^{N}}-1-\sigma_{i j}^{N}\right) .
\end{aligned}
$$

Можно заметить, что в случае $k>m$ мартингальная мера может быть не единственной. Применяя формулы для параметров мартингальной меры (7) и (8) с функцией полезности Выпуск \#1(13), 2010 
$u(x)=x-x^{2} / 2, x<1$, соответствующей минимальной мартингальной мере, получаем, что $\psi_{j} \equiv 1$ и

$$
\begin{aligned}
& v=\sum_{i=1}^{m} \pi_{i} \sigma_{i j}^{W}, \\
& r_{j}=1-\sum_{i=1}^{m} \pi_{i}\left(e^{\sigma_{i j}^{N}}-1\right), \quad r_{j}>0 \\
& \bar{E}_{i}=\sum_{j=1}^{d} \sigma_{i j}^{W} \sum_{l=1}^{m} \sigma_{l j}^{W} \pi_{l}+\sum_{j=1}^{k-d} \lambda_{\mathrm{j}} \sum_{l=1}^{m} \pi_{l}\left(e^{\sigma_{i j}^{N}}-1\right)\left(e^{\sigma_{l j}^{N}}-1\right) .
\end{aligned}
$$

Обозначим через $\Sigma$ матрицу $m$ на $k$ с рядами:

$\left(\sigma^{\mathrm{w}}{ }_{\mathrm{i} 1}, \ldots, \sigma^{\mathrm{w}}{ }_{\mathrm{id}}, \sqrt{\lambda_{\mathrm{j}}}\left(e^{\sigma_{i 1}^{N}}-1\right), \ldots, \sqrt{\lambda_{\mathrm{j}}}\left(e^{\sigma_{i(k-d)}^{N}}-1\right)\right)$.

Решая (11), получим вектор $\pi=\left(\Sigma \Sigma^{t r}\right)^{-1} b \quad$, если $\operatorname{det}\left(\Sigma \Sigma^{t r}\right) \neq 0$.

Относительно минимальной мартингальной меры

$\mathrm{W}^{*}{ }_{\mathrm{j}}(\mathrm{t})=\mathrm{W}_{\mathrm{j}}(\mathrm{t})+\sum_{l=1}^{m} \sigma_{l j}^{W} \pi_{l} t$

будет процессом броуновского движения, а интенсивность пуассоновского процесса будет равна $r_{j} \lambda_{j}$. Обозначим

$$
b_{i}^{*}=\sum_{j=1}^{d} \sigma_{i j}^{W} \sum_{l=1}^{m} \sigma_{l j}^{W} \pi_{l} t
$$

Предположим, что $m=2, d=1$ и $k=2$. То есть в проекте два актива, неопределенности в которых описываются одним броуновским движением и двумя пуассоновскими процессами. Тогда, проводя процедуры, которые используются при выводе формулы БлэкаШолтца, получим, что цену европейского опциона колл $C_{i}=E_{P *}\left(\max \left(S_{i}(T)-K, 0\right)\right)$ можно записать так:

$$
C_{i}=\sum_{j=1}^{2} \sum_{n_{j}=1}^{\infty}\left(e ^ { b _ { i } ^ { * } T + \sum _ { j = 1 } ^ { 2 } \sigma _ { i j } ^ { N } n _ { j } } Ц \left(B_{i}^{+}\left(n_{1}, n_{2}\right)-K \Phi\left(B_{i}^{-}\left(n_{1}, n_{2}\right)\right) \prod_{j=1}^{2} \frac{e^{-\lambda_{j}^{*} T}\left(\lambda_{j}^{*} T\right)^{n_{j}}}{n_{j} !} .\right.\right.
$$

где $Ф$ - функция распределения стандартной нормальной величины, а

$$
B_{i}^{ \pm}\left(n_{1}, n_{2}\right)=\frac{1}{\sigma_{i}^{W} \sqrt{T}}\left(\left(b_{i}^{*} T+\sum_{j=1}^{2} \sigma_{i j}^{N} n_{j}\right) \pm\left(\sigma_{i}^{W}\right)^{2} \frac{T}{2}-\log K\right) .
$$

Таким образом, мы получили формулу, аналогичную формуле Блэка-Шолца. Если k = $\mathrm{m}$ мартингальная мера для (9) будет единственная. Используя формулу (12), можно подсчитать цену реального опциона для минимальной мартингальной меры.

\section{2. Метод Монте-Карло}

Вновь предположим, что $m=2, d=1$ и $k=2$ и активы заданы формулой (2). Пусть скачки маркированных точечных процессов $N_{j}$ распределены экспоненциально с параметром $\beta_{j}$ и ограничены числом 20 (truncated exponential). Предположим также, что $b_{1}=b_{2}=0,05$. Предположим, что все неопределенности в проекте обладают свойством сезонности, т.е. представляют собой синусоиды, а именно $\sigma^{W}{ }_{1}(t)=0,88|\sin (t)| ; \sigma^{W}{ }_{2}(t)=0,84|\sin (t)| ; \sigma_{11}^{N}(t, z)=$ $0,59|\sin (2 t)| ; \quad \sigma^{N}{ }_{12}(t, z)=-0,48|\sin (2 t)| ; \quad \sigma^{N}{ }_{21}(t, z)=0,57|\sin (4 t)| ; \quad \sigma^{N}{ }_{22}(t, z)=-0,44|\sin (4 t)|$. Интенсивность скачков вверх становится больше (процесс с положительными $\sigma^{N}$ ), если цена актива падает ниже определенной границы; и, наоборот, если цена актива поднимается выше некоторого числа, то интенсивность скачков процесса с отрицательными $\sigma^{N}$ вырастает. Можно сказать, что цена актива не может слишком сильно вырасти или упасть. Остальные параметры модели заданы так $T=1, K_{1}=K_{2}=0,2$. Мы разбиваем временной интервал на 200 частей и симулируем 50 раз процессы $S_{i}$, относительно первоначальной меры по формуле 
(2). После преобразования меры в минимальную мартингальную симулируем процессы $Z S_{i}$, где плотность минимальной мартингальной меры $Z$ определяется с помощью формулы (4) с параметрами (8) и функцией полезности $u(x)=x-x^{2} / 2, x<1$. Используя метод Монте-Карло, определяем цену опциона, как

$E\left(Z \max \left(S_{i}(T)-K, 0\right)\right)$.

Получаем следующие значения для цен опционов и вариации цен активов:

Таблица 1

\begin{tabular}{|l|l|l|}
\hline & Первоначальная мера & $\begin{array}{l}\text { Минимальная мартингальная } \\
\text { мера }\end{array}$ \\
\hline $\mathrm{C}_{1}$ & 0,9536 & 0,8483 \\
\hline $\operatorname{Var}\left(S_{1}\right)$ & 0,2178 & 0,0555 \\
\hline $\mathrm{C}_{2}$ & 0,9263 & 0,8348 \\
\hline $\operatorname{Var}\left(S_{2}\right)$ & 0,1461 & 0,0307 \\
\hline
\end{tabular}

Из графиков (см. Приложение 1) и значений вариации в таблице 1 видно, что относительно минимальной мартингальной меры значительно снижаются колебания цен активов, а это приводит к снижению риска, и, следовательно, цены опционов также снижаются. Таким образом, не происходит завышения оценки, традиционного для применения метода реальных опционов в моделях с геометрическим броуновским движением.

\section{Заключение}

Рассмотренные выше примеры показывают, как с помощью мартингального подхода можно определить цены реальных опционов для семимартингальных моделей. Использование формулы (12) или применение метода Монте-Карло, когда для симулирования случайного процесса относительно новой меры достаточно просто умножить исходный процесс на плотность $Z$, значительно упрощает задачу оценки реальных опционов. Для сравнения заметим, что расчет цен опционов путем решения соответствующих дифференциальных уравнений (таких, как уравнение Ито и его обобщения) часто наталкиваются на проблему сходимости численных методов.

Оптимальные мартингальные меры могут быть применены для определения цен реальных опционов на расширение бизнеса или проекта. Можно также отметить использование мартингального подхода в проектах, связанных с энергоэффективностью, как у Ли и Хуан [Lin, Huang, 2009].

Сзолгайова и другие [Szolgayova, Fuss, Obersteiner, 2008] оценивали проект, в котором выбирается источник энергии в условиях регулирования выбросов $\mathrm{CO}_{2}$, с помощью метода реальных опционов. В проекте возникает реальный опцион: либо продолжить закупки электричества, либо построить электростанцию на угле или на биомассе. В этом случае можно разделять два актива. Одним из них является электростанция на угле с денежными потоками, зависящими от возможных ограничений на выбросы $\mathrm{CO}_{2}$ (которые спровоцируют скачкообразное падение стоимости), а также от изменения цен на электричество и уголь. Другим активом может стать электростанция на биомассе. Тогда цены этих активов могут быть смоделированы двумя семимартингалами с несколькими факторами неопределенности. А значит, стоимость опциона на запуск электростанции на угле или на биомассе может быть определена с помощью мартингального подхода.

Таким образом, можно отметить, что семимартингальные модели существенно расширяют возможности применения метода реальных опционов. Если в развитых странах возможно хотя бы приблизительно смоделировать изменения финансовых показателей с помощью геометрического броуновского движения, то в развивающихся экономиках наличие большого количества неопределенностей значительно усложняет задачу поиска 
подходящего процесса. Для учета всех факторов риска при оценке российской компании или проекта недостаточно просто увеличить параметр волатильности в модели Блэка-Шолца, что повлечет за собой рост стоимости соответствующего реального опциона и завышенную оценку. Семимартингальная модель позволяет учесть большую часть факторов риска, а максимизация функции полезности в определении опциона поможет избежать неадекватных оценок. Возможно, применение таких моделей сделает метод реальных опционов более популярным в российской практике.

\section{Список литературы}

1. Ширяев А.Н., Основы стохастической финансовой математики, Стохастика. Т. 2, 3. М.: Фазис, - 1998.

2. Aase, K. (1988), Contingent claims valuation when the security price is a combination of an Ito process and a random point process, Stochastic Processes Applications, 28 (1988) 185220.

3. Bandorf- Nielsen, O.E. (1977), Hyperbolic distributions and distributions on hyperbolae, Proceedings of the Royal Society London, A 353 (1977) 401-419.

4. Barrieu, Bellamy (2007), Optimal hitting time and perpetual option in a non - levy model: application to real options, Adv. Appl. Prob., 39 (2007) 510-530.

5. Black F., Scholes M. (1973), The pricing of options and corporate liabilities, Journal of Political Economy, 81 (1973) 637-654.

6. Bremaud P., Point processes and queues, Springer series in statistics: Springer - Verlag, 1981.

7. Delbaen F., Schachermayer W. (1998), The fundamental theorem of option pricing for unbounded stochastic processes, Mathematische Annalen, 313 (1998) 215-250.

8. Fritelli M. (2000), The minimal entropy martingale measure and the valuation problem in incomplete markets, Mathematical Finance, 10 (2000) 39-52.

9. Follmer H., Schweizer M. (1991), Hedging of contingent claims under incomplete information, in: Davis H. and Elliott R.J. (eds.). Applied Stochastic Analysis, London: Gordon and Breach, p. 389-414.

10. Goll T., Kallsen J. (2000), Optimal portfolios for logarithmic utilities, Stochastic Processes and their Applications, 89 (2000) 31-48.

11. Goll T., Ruschendorf L. (2001), Minimax and minimal distance martingale measures and their relationship to portfolio optimization, Finance and Stochastics, 5 (2001) 557-581.

12. Harrison J. M., Pliska S. (1981), Martingales and stochastic integrals in the theory of continuous trading, Stochastic Processes and their Applications, 11 (1981) 215-260.

13. Jacod J., Shiryaev A.N., Limit theorems for stochastic processes, A series of comprehensive studies in mathematics: Springer - Verlag - 1987.

14. Kallsen J., Shiryaev A.N. (2002), The cumulant process and Esscher's change of measure, Finance and Stochastics, 6 (2002) 397-428.

15. Kallsen J. (1999), A utility maximization approach to hedging in incomplete markets, Math. Meth. of Operations Research, 50(1999) 321-338.

16. Kallsen J. (2002), Derivative pricing based on local utility maximization, Finance and stochastics, 6 (2002) 115-140.

17. Kumbaroğlu, Madlener R., Demirel M. (2008), A real options evaluation model for the diffusion prospects of new renewable power generation technologies, Energy Economics, 30 (2008) 1882-1908.

18. Lin T., Huang S.L. (2009), An entry and exit model on the energy- saving investment strategy with real options, Energy Policy, 3 (2009) 32-39.

19. Merton R. (1973), Theory of rational option pricing, Bell Journal of Economics and Management Science, 4 (1973) 141-183. 
20. Pyo U. (2008) Real option pricing and bounds in incomplete markets. The Engineering Economist, Jan. - Mar. (2008) 53.

21. Trigeorgis L.. Real Options: Managerial Flexibility and Strategy in Resource Allocation Cambridge, MA.: MIT Press, -1996

22. Samuelson P. (1965), Rational theory of warrant pricing, Industrial management review, 6 (1965) 13-32.

23. Schweizer M. (1996), Approximation pricing and the variance optimal martingale measure, The Annals of Probability, 24 (1996) 206-236.

24. Staum J. (2007), Incomplete Markets, Handbooks in Operations Research and Management Science, 15, (2007) 511-563.

25. Szolgayova J., Fuss S., Obersteiner M. (2008), Assessing the effects of CO2 price caps on electricity investments - A real options analysis, Energy Policy, 36 (2008) 3974-3981. 


\section{Приложение 1}

Рисунок 1. S1 относительно первоначальной меры.

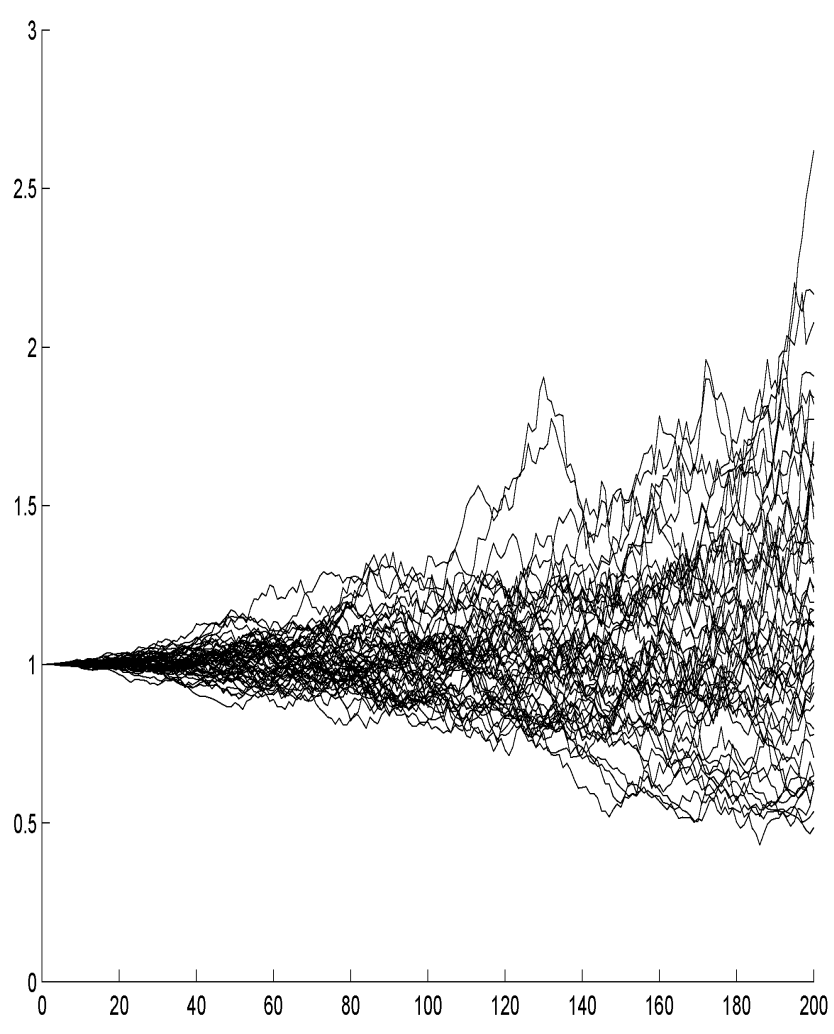

Рисунок 3. Гистограмма цены опциона для меры $P$

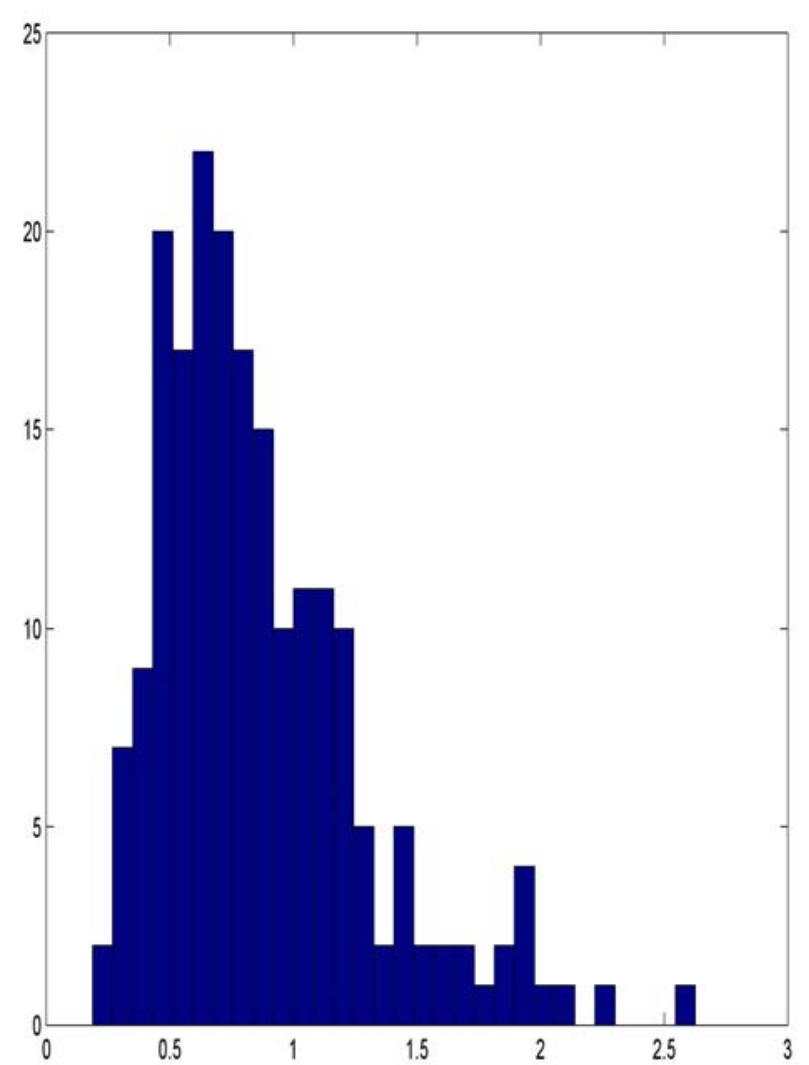

Рисунок 2. $\mathrm{S}_{1}$ относительно минимальной мартингальной меры.

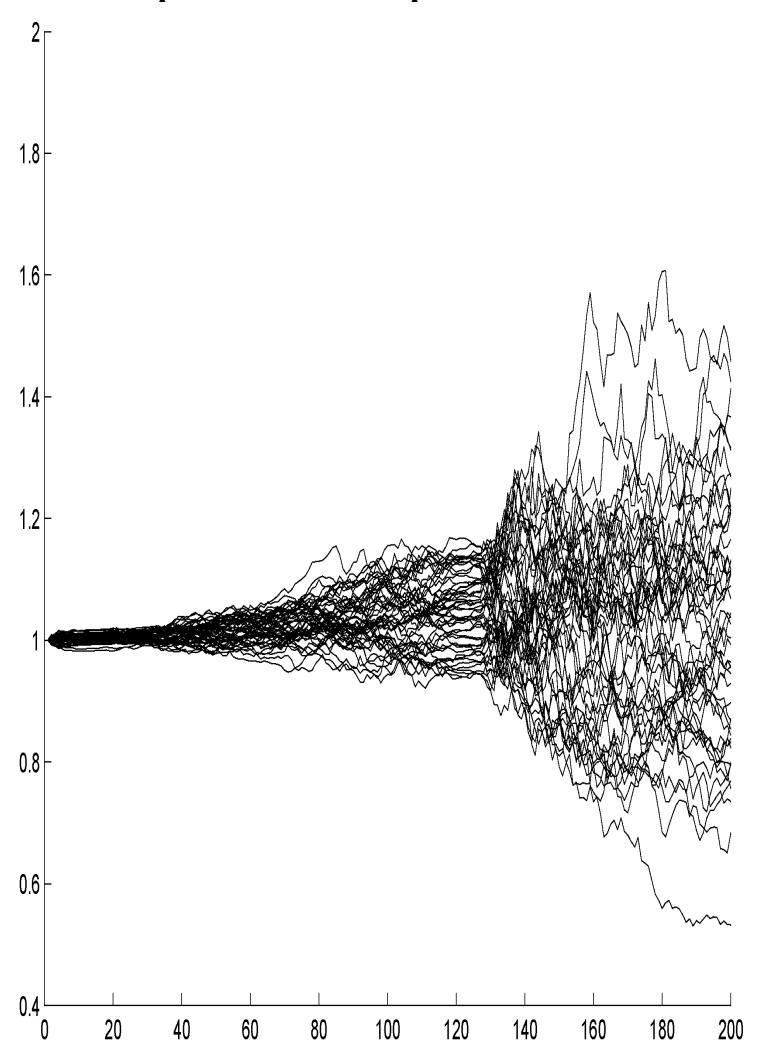

Рисунок 4. Гистограмма цены опциона для меры $P^{*}$

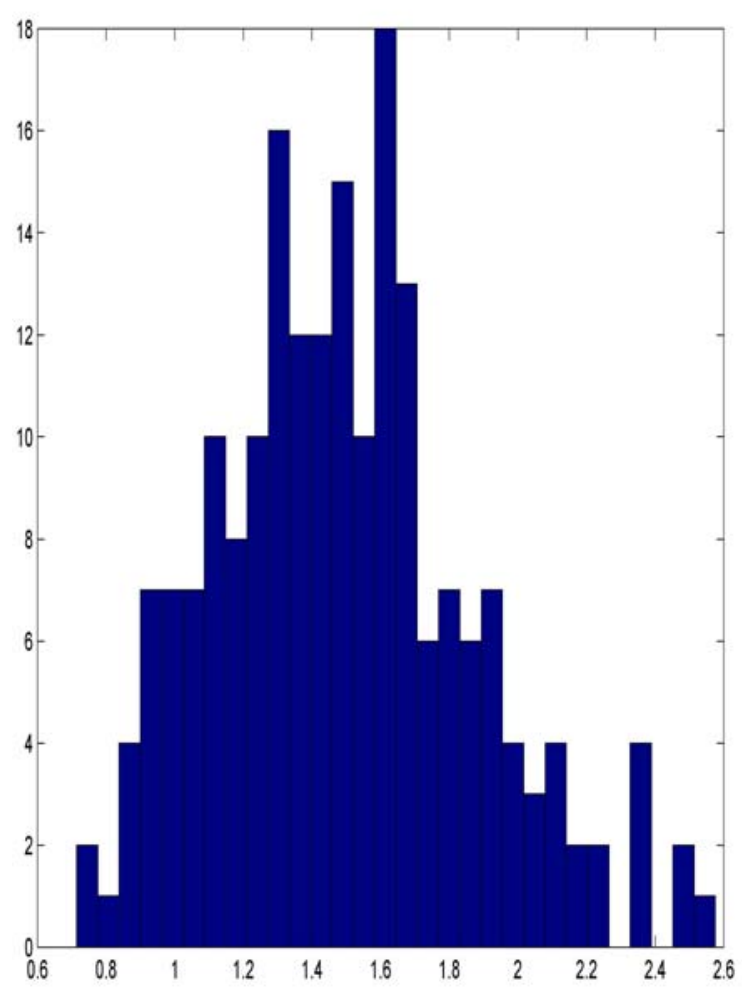

Выпуск \#1(13), 2010

() Электронный журнал Корпоративные Финансы, 2010 